IJPP 2009, 17: 269-274 (C) 2009 The Authors Received June 10, 2008 Accepted February 03, 2009 DOI 10.1211/ijpp/17.05.0003 ISSN 0961-7671

\section{Development of protocols for the provision of headache and back-pain treatments in Maltese community pharmacies}

\author{
Elaine Vella, Lilian M. Azzopardi, Maurice Zarb-Adami \\ and Anthony Serracino-Inglott
}

Department of Pharmacy, University of Malta, Msida, Malta

\begin{abstract}
Objective The purpose of this study was to draw up two protocols designed to help Maltese pharmacists care for consumers seeking treatment for headache and back pain and to subsequently use the protocols to assess pharmacists' management of the named conditions. Method The setting was a sample of 10 of the 207 community pharmacies in Malta. Two flow-chart protocols for headache and back-pain management were developed from various reference sources. The protocols were first tested in a community pharmacy for practicality and applicability in a pilot study. In nine other pharmacies chosen at random the pharmacists' manner of addressing 10 headache and 10 back-pain cases in each pharmacy was compared with that recommended in the protocols. Consumers who visited the pharmacy to fill a prescription, to purchase a named product or for advice on how to deal with symptoms were
\end{abstract} included in the study.

Key findings Of the 212 pharmacist interventions assessed, cases where pharmacists responded to symptoms were managed with the highest average compliance (57\%) whereas cases in which the consumer asked for a product by name were managed with an average compliance with the protocols of $46 \%$. Cases in which consumers presented at the pharmacy with a prescription were managed with an average compliance of $55 \%$.

Conclusions Protocols may be used as a means of measuring the impact of the intervention of community pharmacists in patient care. The findings suggest a lack of advice given to consumers presenting at the pharmacy to request a named product.

Keywords back pain; community pharmacy; headache; protocols

\section{Introduction}

The intervention of community pharmacists in responding to consumers with minor ailments continues to be one of the major contributions by community pharmacists to the maintenance of the best health possible for their society ${ }^{[1]}$ The demand for primary care in a community pharmacy setting is on the increase ${ }^{[2-4]}$ and is associated with a number of factors, including the need for medicines, patient perceptions of pharmacists' healthcare role and the nature of the presenting condition; indeed, symptoms of minor self-limiting conditions are usually given as the main reason for using a pharmacy. ${ }^{[5]}$

With more than $90 \%$ of the population experiencing one or more headaches in their lifetime ${ }^{[6,7]}$ and approximately $80 \%$ of adults experiencing low back pain that will affect their daily activities at some point in their lives, ${ }^{[8]}$ the community pharmacist is often approached with regards to advice for and treatment of these conditions. ${ }^{[9]}$ With $66 \%$ of patients who take medications for headache actually buying these from a pharmacy ${ }^{[10]}$ and back pain identified as one of the most common complaints pharmacists hear from patients seeking advice and over-the-counter pain relief, ${ }^{[11,12]}$ self-medication seems to be the main method of treatment. Headache and back pain were thus considered ideal topics for protocol development since pharmacists are in an ideal place to provide advice for these conditions and help improve patient care.

The identification and addressing of patients' needs and concerns by community pharmacists has, however, been shown to be variable. ${ }^{[13,14]}$ With protocols, pharmacists are assisted in the decision-making process of responding to symptoms, recommended treatment choices are evidence-based and patient outcomes improved. ${ }^{[15,16]}$ A protocol is defined as a disease-based therapeutic algorithm, which begins with a confirmed diagnosis of a particular
Zammit Clapp Hospital,

Pharmacy Department,

St Julians, GZR 1540, Malta.

E-mail: elaine.vella@gov.mt 
disease and directs the healthcare provider through a series of decisions that differentiate patients into alternative pathways. $^{[17]}$

Notwithstanding the advantages of protocols, little work on their development and use has been carried out in Malta. In Malta, the availability of over-the-counter medicines is limited to pharmacies and no other retail outlet. There is on average one community pharmacy for every 2000 persons. Pharmacies have only one pharmacist on duty at a particular time. Other pharmacists replace the managing pharmacist on a part-time basis. This results in $44 \%$ of Maltese pharmacists contributing to the profession through pharmacy practice. ${ }^{[18]}$ Seventy seven per cent of pharmacies employ one or more pharmacy technicians or salespersons or both. ${ }^{[1]}$ There is no requirement for salespersons to hold a minimum qualification to work in pharmacies. Pharmacy technicians may dispense medicinal products only under the supervision of a pharmacist.

The purpose of this study was thus to develop flow-chart headache and back-pain protocols to be used in the community pharmacy setting. The protocols were intended to act as a guideline by highlighting a specific plan according to which the condition of the patient was best managed. The protocols were subsequently used in a pilot study to test their practicality and applicability in the community pharmacy setting. A final goal was to use the protocols as a means of evaluating pharmacists' interventions according to the manner in which patients were questioned, advice given and treatment recommended.

\section{Method}

\section{Protocol design}

Two flow-chart protocols, for headache and back-pain management, were developed using key pharmacy journals, standard textbooks and other references. The format selected for the presentation of the protocols was that used by the American Pharmaceutical Association (APhA), ${ }^{[17]}$ which translates the information into a flow chart, deemed ideal for succinctly conveying the relationship and sequential direction of steps. Each protocol included the following. (1) Questions about patient identity as well as the duration, location, intensity and type of pain: this type of specific information about the person requiring treatment is essential for the pharmacist. (2) A onepage diagram, termed the prescription sheet, which contained the series of steps to be followed when the pharmacist was presented with a prescription. (3) Statements detailing the most encountered headache or back-pain conditions. These statements were designed to distinguish between the different conditions on the basis of the different accompanying symptoms and trigger factors. For example, the constricting band of pain felt around the head when a patient is under stress, typical of a tension headache, helps to differentiate it from the dull pain and tenderness around the eyes and cheekbones accompanied by a blocked nose, typical of sinusitis. ${ }^{[19,20]}$ The diagnosis ends with a decision of whether to treat or refer the patient. (4) A treatment sheet which outlined the management of the condition, including non-pharmacological measures and over-the-counter pharmacological treatment. (5) An explanatory text, the main purpose of which was to present the background information and references to support information in the flow charts.

\section{Evaluation of the protocols}

Qualitative validity, applicability and practicality testing were employed. To assess validity of the protocols, two general practitioners and two pharmacists were approached. The aims of the study were outlined. Meetings were held with each to review content validity of the protocols. The length of the protocol was questioned; however, it was agreed to retain all areas so as to present a comprehensive overview of both conditions. Applicability and practicality of the protocols were assessed through a pilot test. The researcher visited one community pharmacy, chosen for convenience, to ascertain that the protocols were acceptable for use in the practical setting and that it was feasible to use the protocols to assess pharmacists' interventions through observation. The pilot study also helped determine whether 10 cases were likely to be collected within the set time frame and how long data collection was likely to take. The documentation form was also reviewed to check its adequacy for recording the necessary information. Results from this pharmacy were included in the final analysis since the minor changes made to the protocols still permitted the grouping of data.

\section{Assessing pharmacists' interventions}

For the assessment of pharmacists' interventions using the protocols, the non-participant observation technique was adopted since it is simple and feasible to perform within the environment of a community pharmacy when compared to other documentation techniques. ${ }^{[1]}$ It does not rely on a consumer's disposition to respond to questions or ability to recall events (as opposed to self-completed questionnaires), and avoids eligible consumers not participating during times when the pharmacy is particularly busy or short of staff (reasons for non-recruitment by pharmacists). ${ }^{[21]}$

Nine pharmacies were chosen at random (from a list of all pharmacies in Malta), in which to perform the research. This brought the total number of pharmacies to 10 since pilot data collected from the first pharmacy were also included in the final analysis. The managing pharmacists were contacted and informed of the aims of the study. The dates and times of each observation session were agreed.

During the first observation session in each pharmacy, pharmacists went through the protocols and gave their feedback. Each pharmacy was then attended for the length of time required to document pharmacists' interventions in 10 headache and 10 back-pain cases. A record was also kept of the total number of consumers walking into the pharmacy during the observation sessions.

Consumers visiting a pharmacy have a number of options when presenting with a minor condition. ${ }^{[22]}$ For the purposes of this study, all pharmacist interventions were placed in three categories. Cases where consumers presented at the pharmacy to fill a prescription were termed Prescription cases. Those cases where consumers requested a product by name were classified as Specific product cases and those cases where consumers frequented the pharmacy for advice on how to deal with symptoms were designated as Describing symptoms cases. The inclusion criterion for these three 
categories was that there had to be some exchange of information between consumer and pharmacist, allowing the observer to identify the case as being pertinent to the study.

Data for each case were recorded on a documentation form. In the first column, the observer ticked the steps from the protocol which were followed by the pharmacist during the management of a particular case. In a second column, the observer ticked all the steps which should have been followed according to the protocol. By comparing the divergence between the two sets of data the pharmacist's compliance with the protocol for each case was calculated. For the purpose of the study compliance was defined as 'a measure of the extent to which the pharmacist's behaviour matched the recommendations in the protocols'. ${ }^{[23]}$

Ethical approval was not required for the purposes of this study, since no patient data were recorded. Only pharmacists who consented to participate in the study and their management of headache and back-pain cases were observed.

\section{Results}

\section{Response rate and demographics}

Nine pharmacists out of the 10 contacted agreed to take part in the study. One pharmacist refused to participate, claiming restrained space in the pharmacy. Another pharmacy was chosen at random instead. Of the pharmacies recruited to take part in the study, three were in the Southern Harbour district, two in the Northern Harbour district, one in the Western District, and four in the Northern District. Two districts - the South-Eastern District and Gozo (geographically isolated) were not represented. The mean population per pharmacy was 1912. In these pharmacies only one pharmacist was in attendance at any one time. Salespersons were absent in three pharmacies, all three owned by a pharmacist. Five pharmacies had one salesperson and two pharmacies had two and three salespersons respectively. An estimated 21 consumers per hour visited the pharmacies.

\section{The protocols}

This study set out to draw up two protocols based on two conditions commonly present in primary care: headache and back pain. One hundred and fourteen steps were set up to diagnose and manage the different headache conditions while 89 steps made up the back-pain protocol. Figure 1 shows an excerpt from the headache protocol. In Box 1, a consumer presents at the pharmacy with a complaint of headache. Box 2 establishes whether the pharmacist is familiar with the consumer, in which case the pharmacist is directed to Box 4 . If the person presenting at the pharmacy is a new consumer, the pharmacist is directed to Box 3 where questions are asked about the patient's identity. In Boxes 4 and 5 the pharmacist asks about the duration and location of pain. These boxes refer the pharmacist to the explanatory text where more detail can be found.

\section{Validity, applicability and practicality}

Content validity of the two protocols was found to be strong. Statements in the protocol were clear and comprehensive. During the pilot test, the protocols were found to be: detailed

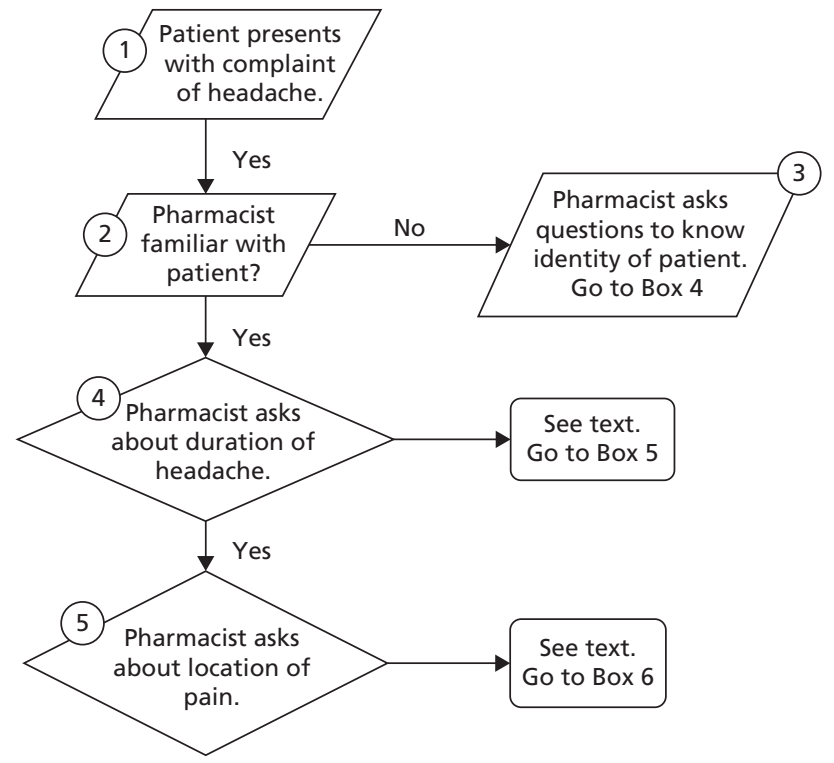

Figure 1 Excerpt from the headache protocol

and comprehensive to diagnose and manage the different headache and back-pain conditions encountered in the community pharmacy setting; uncomplicated to follow with an evident layout of information flow; and able to be used as a means of assessing pharmacists' interventions in the management of headache and back-pain disorders.

\section{Interventions}

A total of 212 pharmacist interventions were assessed in the 182 observation hours spent at the 10 participating pharmacies. All cases were managed with an average compliance of $52 \%$ (range 6-100\%). Figure 2 graphically portrays the percentage compliance of all the recorded cases with the respective protocols.

Describing symptoms cases accounted for almost half of the total cases $(42 \%, n=90$ cases). They were managed with an average compliance of $57 \%$ (range 6-100\%). Prescription cases ( $n=63$ cases) made up a further $30 \%$ of the total. They were managed with an average compliance of 55\% (range 29-94\%). Specific product cases ( $n=59$ cases) were the least encountered, with $28 \%$ of the total cases. They were managed with an average compliance of $41 \%$ (range 10-90\%).

\section{Headache protocol}

The average compliance for the 109 headache cases was $52 \%$ (range 6-100\%).

Describing symptoms cases ( $n=57$ cases) accounted for more than half of the total headache cases. They were managed with an average compliance of $56 \%$ (range 6-100\%). Only one case was managed with $100 \%$ compliance to the headache protocol. There were 18 cases managed with a compliance higher than $70 \%$.

Headache Prescription cases were the least encountered, making up $20 \%$ ( $n=22$ cases) of all headache cases. The average compliance was $53 \%$ (range 29-75\%). Only five cases were managed with a compliance higher that $70 \%$. 


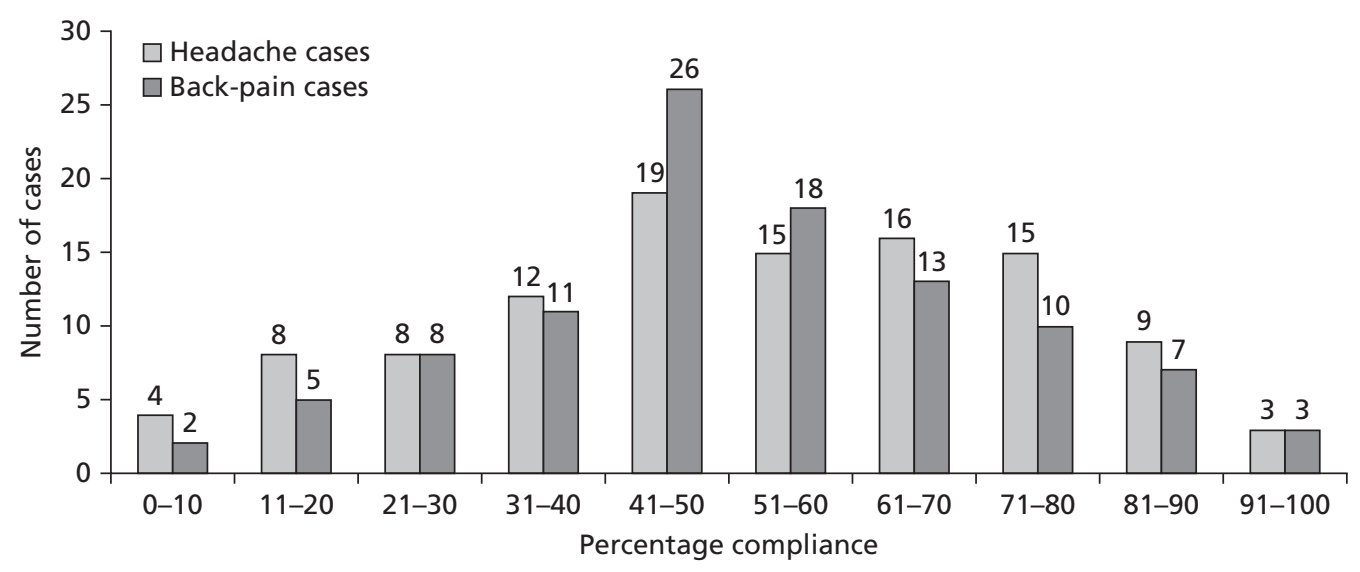

Figure 2 Graph showing the percentage compliance of headache and back-pain cases with the respective protocols. Numbers above each bar indicate the number of cases.

The $28 \%$ ( $n=30$ cases) of headache cases classified as Specific product cases had the lowest average compliance of $46 \%$ (range 14-90\%). Only four cases were managed with a compliance higher than $70 \%$.

The most commonly presented headaches were those as part of cold symptoms, which added up to $19 \%$ of all cases ( $n=21$ cases). These were followed by tension headaches ( $n=18$ cases $)$, sinus headaches $(n=16$ cases) and migraine headaches ( $n=15$ cases). There were four cases in which the headache was thought to be drug-induced. In $11 \%$ of cases ( $n=12$ cases) the pharmacist did not establish the type of headache or the circumstances that caused it. These were mostly cases where the consumer requested a named product.

The most commonly dispensed medications were paracetamol, ibuprofen and combination products, which contained paracetamol, ibuprofen, aspirin, caffeine, codeine (up to $12.5 \mathrm{mg}$ per tablet), antihistamines and nasal decongestants.

\section{Back-pain protocol}

The average compliance for the 103 back-pain cases collected was $52 \%$ (range 10-94\%).

The $32 \%$ ( $n=33$ cases) of back-pain cases placed in the Describing symptoms category had an average compliance of 59\% (range 10-93\%). There were 13 cases managed with a compliance higher than $70 \%$.

Back-pain Prescription cases were the most encountered, making up $40 \%$ ( $n=41$ cases) of all back-pain cases. The average compliance was 57\% (range 33-94\%). Only six cases were managed with a compliance higher that $70 \%$.

The $28 \%$ ( $n=29$ cases) of back-pain cases placed in the Specific product category had the lowest average compliance of $37 \%$ (range $10-81 \%$ ). Only one case was managed with a compliance higher than $70 \%$.

The most commonly presented type of back pain was that associated with acute muscle strain ( $n=39$ cases). Patients complained of lifting too heavy objects, lifting objects improperly and imprudent exercising. The second most common type of back pain was non-specific back pain ( $n=10$ cases). In most of these cases the consumer would have had the pain before, it reoccurred, but the consumer could not pinpoint the cause of the back pain. In $29 \%$ of cases $(n=30)$, the pharmacist did not establish the type of back pain or the circumstances that caused it, mostly cases in which the consumer presented with a prescription.

A topical analgesic was the most commonly dispensed firstline treatment, both on its own or together with an oral analgesic, usually a combination product. Topical non-steroidal antiinflammatory drugs classified as non-prescription items were the most frequently dispensed agents of all.

\section{Compliance to individual steps in the headache and back-pain protocol}

Average compliance to the steps in the first part of each protocol - establishing patient identity and nature of symptoms - was $43 \%$ for the headache protocol (range 7-100\%) and $42 \%$ for the back-pain protocol (range 4-100\%). Average compliance to the prescription sheet was $48 \%$ for the headache protocol (range $0-100 \%$ ) and $63 \%$ for the back-pain protocol (range $0-100 \%$ ). A step with $100 \%$ compliance in this section was one in which the pharmacist was required to explain the dose and dosage regimen of a prescribed medication. Average compliance to the steps containing the statements detailing particular headache or back-pain conditions was $25 \%$ for the headache protocol (range 0-92\%) and 23\% for the back-pain protocol (range $0-76 \%$ ). A step with $0 \%$ compliance in this section was one which requested referral when migraine symptoms occurred for the first time. Average compliance to the treatment sheet was $42 \%$ for the headache protocol (range $0-98 \%$ ) and $33 \%$ for the back-pain protocol (range 0-100\%).

\section{Discussion}

All outcomes devised for this study were achieved. The main objective was the development of two protocols which were well received and generated positive comments from the participating pharmacists. The assessment of pharmacist interventions was then carried out through their compliance with the protocols, rather than through evaluation of patient outcomes.

The average compliance with which all cases were managed was $52 \%$. This indicated that there was not an 
acceptable standard of pharmacist involvement in the management of cases observed. Pharmacists should always be available to answer questions about medications, be accessible when advice is sought and give consumers directions on how to take their medications even when unsolicited. ${ }^{[24]}$ Cases where pharmacists responded to symptoms were managed with the highest average compliance followed by cases in which consumers presented at the pharmacy with a prescription. Cases in which the consumer asked for a product by name were characterized by the least interaction between pharmacist and consumer.

It is important to recognize the limitations of the study. A change in behaviour resulting from awareness of being observed is termed the Hawthorne effect. This could have led to the recruited pharmacists performing better than if they were not being observed. The compliance results might therefore be overestimated. It was attempted to counteract this with repeated visits to each pharmacy by the same observer, so that acute awareness of the presence of the observer would lessen. Also it was imperative that the pharmacists' attention was not drawn to the observer each time a record was taken since the success of observations has been identified as depending on how unobtrusively the observations are performed. ${ }^{[25]}$ Also, the pharmacists were assured anonymity so that they could feel comfortable behaving in their usual way. Another issue involved cases where the consumer asked for a specific product but there was no interaction between pharmacist and consumer. Although these were not included in the study, it is recognized that some of them may have been eligible. Another limitation was the small number of pharmacists recruited to take part in the study. This was also reflected in the small differences in compliance between pharmacists. Five pharmacists fell into the $41-50 \%$ average compliance range, two in the $51-60 \%$ range and three in the $61-70 \%$ range. Only one pharmacist made the $71-80 \%$ average compliance range when dealing with headache cases. None of the pharmacists had an average compliance higher than $69 \%$ when dealing with back-pain cases. However, it was not the authors' intention to identify differences between pharmacists but rather to perform an analysis of the profession. Undoubtedly, increased numbers of pharmacists and consumers would benefit the study if it were to be repeated. At the time of the study ethical approval was not essential for the data collection required. However, this varies according to the nature and setting of the study.

The majority of consumers who presented at the pharmacies with a headache did so to ask for advice or describe symptoms rather than to purchase a named product or fill a prescription. In contrast, consumers who presented with back pain did so mostly to obtain a prescribed product rather than to ask for advice or for a specific product. The fact that consumer behaviour varies according to the nature of the condition has been reported in previous work. ${ }^{[22]}$

Prescription cases were managed with a low compliance with the respective protocol, especially considering the smaller amount of steps that had to be followed with respect to other categories. By not asking questions about the patient's condition pharmacists omit giving timely advice to patients and reinforcing advice given by other healthcare professionals. ${ }^{[14]}$ Previous work confirms that interactions between pharmacists and consumers who obtain treatment on prescription rarely involve questioning and advice from pharmacists, and that certain consumers would neither expect nor want this type of interaction. ${ }^{[26,27]}$

Specific product cases were managed with the lowest average compliance with the protocols. A similar finding was reported by John et al. in their study in Scotland and Wales, to identify factors affecting pharmacist advice. ${ }^{[21]}$ They concluded that consumers requesting a named product were less likely to be advised on the use of the product than those who do not. In fact, pharmacists presume that consumers expect to make over-the-counter purchases without being questioned ${ }^{[28]}$ and feel less obligation to give advice to patients who do not demand it. However, in such cases it is not known whether consumers may have bought products that were inappropriate for their condition or less effective than alternatives. A number of reasons for non-compliance with protocols are reported in the literature, including a fear of consumers' resentment to questioning, a busy pharmacy schedule, time constraints and previous use of the product by the consumer. ${ }^{[19,29,30]}$

Both headache and back-pain Describing symptoms cases were managed with the highest average compliance with the protocols. This reflected a major effort from pharmacists in the management of such cases, rather than when consumers obtained treatment either on prescription or by self-purchase. The fact that more than a third of cases in this category were managed with a compliance higher than $70 \%$ was considered as proof of the relevance of the steps included in the protocols. Morris et al. stated that patients wish to use pharmacies in the way they desire: they want a pharmacist to be available to give advice if they perceive it necessary. ${ }^{[28]}$ Thus, in our study patients who sought advice for the management of their symptoms got the highest degree of interaction with the pharmacist. In this scenario pharmacists followed the protocols and provided a better degree of intervention as expected by the patients who sought this type of professional pharmacist advice.

This study adds to our understanding of how pharmacists respond in relation to presentation of symptoms, requests for specific products and when presented with a prescription. It can serve as a benchmark for Maltese pharmacists to improve their intervention in patient care.

\section{Conclusions}

Protocols can be used to measure the impact of the intervention of community pharmacists in patient care. The developed protocols were found to be applicable to the Maltese community pharmacy setting. The study results indicate a lack of pharmacist involvement in the management of cases, especially cases where consumers asked for a product by name. More emphasis should be placed on the provision of advice to consumers presenting at the pharmacy.

\section{Declarations}

\section{Conflict of interest}

The Author(s) declare(s) that they have no conflicts of interest to disclose. 


\section{Funding}

This research received no specific grant from any funding agency in the public, commercial or not-for-profit sectors.

\section{References}

1. Azzopardi LM. Validation instruments for community pharmacy. Pharmaceutical Care for the Third Millennium. New York: Pharmaceutical Products Press, 2000. pp. 15, 31, 36.

2. Watson MC et al. Educational strategies to promote evidencebased community pharmacy practice: a cluster randomized controlled trial. Fam Pract 2002; 19: 529-536.

3. Bond CM, Watson MC. The development of evidence-based guidelines for over-the-counter treatment of vulvovaginal candidiasis. Pharm World Sci 2003; 25: 177-181.

4. Watson MC, Bond CM. Evidence-based guidelines for nonprescription treatment of vulvovaginal candidiasis. Pharm World Sci 2003; 25: 129-134.

5. Hassell $\mathrm{K}$ et al. Community pharmacy as a primary health and self-care resource: a framework for understanding pharmacy utilization. Health Soc Care Community 2000; 8: 40-49.

6. Glover C. Guidelines for community pharmacists on the management of headache. Pharm J 2008; 280: 311-318.

7. Boardman HF et al. Headache experiences and medication use in primary care. Int J Pharm Pract 2001; 9(suppl.): R34.

8. Warfield CA, Fausett HJ. Manual of Pain Management, 2nd ed. Philadelphia, PA: Lippincott Williams and Wilkins, 2002.

9. Edwards C, Stillman P. Minor Illness or Major Disease? Responding to Symptoms in the Pharmacy, 3rd ed. London: Pharmaceutical Press, 2000.

10. Boardman HF et al. Headaches, self-care and health care seeking behaviour: a pilot study. Pharm J 2000; 265: R36.

11. Pray WS. What to suggest for low back pain. US Pharm 1998; 23.

12. Dickson J. Promoting self care of joint pain. Pharm J 2006; 277 : 285-288.

13. Westerlund $\mathrm{T}$ et al. Evaluation of a model for counselling patients with dyspepsia in Swedish community pharmacies. Am J Health-Syst Pharm 2003; 60: 1336-1341.

14. Silcock J et al. Do community pharmacists have the attitudes and knowledge to support evidence based self-management of low back pain? BMC Musculoskelet Disord 2007; 8: 10.

15. Mohan N, Nagavi BG. Development of standard therapeutic guidelines for selected common diseases for the primary health care centres of a South Indian district. Int J Pharm Pract 2003; 11: $169-174$.

16. Al-Eidan FA et al. Use of a treatment protocol in the management of community-acquired lower respiratory tract infection. J Antimicrob Chemother 2000; 45: 387-394.

17. American Pharmaceutical Association. Drug Treatment Protocols. Washington DC: APhA Publications, 1999. pp. xi-xiii.

18. Mizzi C. A Directory of Pharmacists. Malta: Department of Pharmacy, University of Malta, 2005.

19. Blenkinsopp A, Paxton P. Symptoms in the pharmacy. A Guide to the Management of Common Illness. Oxford: Blackwell Science, 1995. pp. 1-3.

20. Cailliet R. Head and Face Pain Syndromes. Philadelphia, PA: FA Davis Company, 1992. pp. 56-57.

21. John DN et al. Are customers requesting medicines by name less likely to be advised or referred? Provision of over-thecounter H2-receptor antagonists and alginate products from pharmacies. Int J Pharm Pract 2003; 11: 33-39.

22. Seston $\mathrm{L}$ et al. Variation in the incidence, presentation and management of nine minor ailments in community pharmacy. Pharm J 2001; 266: 429-432.

23. Horne $\mathrm{R}$ et al. Concordance, Adherence and Compliance in Medicine Taking. Report for the NCCSDO. 2005. www.sdo.nihr. ac.uk/files/project/76-final-report.pdf (accessed 1 July 2009).

24. Roller K. Consumers give pharmacists high marks as complexity of profession grows. Drug Store News, 1999. http://findarticles. com/p/articles/mi_m3374/is_9_21/ai_54944149 (accessed 1 July 2009).

25. European Design for all e-Accessibility Network. Direct Observation - Tools and Techniques. www.education.edean. org/pdf/Tool054.pdf (accessed 2 July 2009).

26. Blenkinsopp A, Hassey A. Effectiveness and acceptability of community pharmacy-based interventions in type 2 diabetes: a critical review of intervention design, pharmacist and patient perspectives. Int J Pharm Pract 2005; 13: 231-240.

27. Sinclair $\mathrm{H}$ et al. Community pharmacy provision of allergic rhinitis treatments: a longitudinal study of patient reported outcome. Int J Pharm Pract 2005; 13: 249-256.

28. Morris CJ et al. One simple question should be enough: consumers' perceptions of pharmacy protocols. Int J Pharm Pract 1997; 5: 64-71.

29. Bissel $\mathrm{P}$ et al. Insights into the implementation of in-store protocols in community pharmacies. Pharm J 1997; 259: R36.

30. Haynes A. Sale of medicines in pharmacies. Protocols: where now? Pharm J 1997; 259: 66-67. 